\title{
A novel behavioral model of the pasture-based dairy cow from GPS data using data mining and machine learning techniques
}

\author{
M. L. Williams, ${ }^{*}$ N. Mac Parthaláin, † P. Brewer,ł W. P. J. James, ${ }^{*}$ and M. T. Rose ${ }^{\star 1}$ \\ *Institute of Biological, Environmental and Rural Science, Aberystwyth University, Penglais Campus, Ceredigion, SY23 3DA, United Kingdom \\ †Department of Computer Science, Institute of Maths, Physics and Computer Science (IMPACS), and \\ ‡Department of Geography and Earth Sciences, Aberystwyth University, Penglais Campus, Ceredigion, SY23 3DB, United Kingdom
}

\section{ABSTRACT}

A better understanding of the behavior of individual grazing dairy cattle will assist in improving productivity and welfare. Global positioning systems (GPS) applied to cows could provide a means of monitoring grazing herds while overcoming the substantial efforts required for manual observation. Any model of behavioral prediction using GPS needs to be accurate and robust by accounting for inter-cow variation as well as atmospheric effects. We evaluated the performance using a series of machine learning algorithms on GPS data collected from 40 pasture-based dairy cows over 4 mo. A feature extraction step was performed on the collected raw GPS data, which resulted in 43 different attributes. The evaluated behaviors were grazing, resting, and walking. Classifier learners were built using 10 times 10-fold cross validation and tested on an independent test set. Results were evaluated using a variety of statistical significance tests across all parameters. We found that final model selection depended upon level of performance and model complexity. The classifier learner deemed most suitable for this particular problem was JRip, a rule-based learner (classification accuracy $=0.85$; false positive rate $=0.10 ; \mathrm{F}$-measure $=0.76$; area under the receiver operating curve $=0.87$ ). This model will be used in further studies to assess the behavior and welfare of pasture-based dairy cows.

Key words: GPS, grazing, behavior model, data mining

\section{INTRODUCTION}

It is well documented that the behavior of dairy cattle coincides with changes in health status. For example, Huzzey et al. (2007) used electronic feed bins

Received August 14, 2015.

Accepted November 28, 2015.

${ }^{1}$ Corresponding author: mir@aber.ac.uk to record feeding behavior in housed dairy cows. They found that feed intake and time spent feeding began to decrease 2 wk before clinical diagnosis of severe metritis. González et al. (2008) found that daily feeding time, number of visits to the feed bin, and feeding rate began to decrease as early as $30 \mathrm{~d}$ before lameness diagnosis in housed cows fed a silage ration. With larger herds and limited time, disease diagnosis becomes more difficult. Mobility scoring is one example of a subjective technique used by herdsmen to identify lameness and locomotor problems in dairy cows. Although cheap to disseminate, mobility scoring is time consuming and must be done regularly (Pluk et al., 2012; Van Nuffel et al., 2015). Another criticism of the technique is that it may fail to identify the early (sub-clinical) stages of lameness (Manske et al., 2002; Dyer et al., 2007). Reader et al. (2011) reported that the milk yield of cows decreased by an average of $0.7 \mathrm{~kg} / \mathrm{d}$ for approximately 7 wk before cows became visually lame. Furthermore, after recovery, the milk yield of lame cows remained lower for 4 wk. Identifying production disease as early as possible is therefore imperative to minimize welfare implications and production loss. Technology designed to identify lame cows using pressure plates to measure weight distribution, for example, has already been assessed (Bicalho et al., 2007). Although they concluded that more work was needed to refine the sensitivity of these devices, weight shifting by the cow may be visible by gait assessment and therefore these tools are likely to be effective in reducing the labor cost of mobility scoring. An approach to identify sub-clinical disease possibly by using behavioral changes before gait abnormalities are present may be more constructive.

Technology available for domestic and other commercial applications can be used to improve dairy cattle welfare and performance. For example, pedometers are effective in detecting increased activity during estrus, thus aiding dairy cattle fertility management (Roelofs et al., 2005). However, there is little scope for cattle behavioral classification using pedometer data. Accelerometers, on the other hand, have provided the ability 
to identify lying and standing behavior in grazed dairy cattle (Munksgaard et al., 2006; Nielsen et al., 2010). Nielsen (2013), using a 3-dimensional head-mounted activity logger to identify grazing behavior, achieved a true positive classification rate of $84 \%$. Dutta et al. (2015) achieved an average classification accuracy of $93 \%$ for grazing, searching, ruminating, resting, and scratching when using a collar-mounted unit consisting of a global positioning system (GPS) receiver and 3 -axis accelerometer. A behavioral recording system needs to be robust to the cow's environment and as accurate as possible. Pedometers attached to legs are open to damage and head-mounted accelerometers could be laborious to apply to cows. Furthermore, a system needs to be able to identify the main behaviors of the pasture-based dairy cow, preferably as a single, discrete, and lightweight unit that is cheap to deploy in a commercial environment. As such, the scope for utilizing GPS collars alone to collect frequent temporal and positional data seems attractive.

The application of data mining and machine learning techniques in livestock behavioral studies to search for patterns in data that are unobservable by the human eye has been limited until recently. This has largely been because collecting a vast amount of behavioral data has been difficult. Such techniques have already been used to mine cattle disease databases to identify herd disease risk, for example (Ortiz-Pelaez and Pfeiffer, 2008), and to make breeding decisions based on the likelihood of conception from previous insemination data and disease history (Shahinfar et al., 2014). Applying these techniques to behavioral data could present opportunities to learn more about the subtle changes that may occur over time during the onset of disease. The use of small, high data acquisition GPS receivers that are cheap and easy to apply to cattle may be a good incentive for pasture-based dairy practitioners provided the data are accurate and reliable. Furthermore, positional data could be extended to supply information on energy expenditure and pasture preference for grazing management as well as monitoring health status over time.

As far as we are aware, no published model of the main behaviors of pasture-based dairy cows from GPS data currently exists. The objective of this study was 2 -fold. The first was to assess the ability of machine learning techniques in identifying the main behaviors grazing, resting, and walking from GPS data. The second objective was to gain information about how these behaviors are represented within GPS data and to fully document this behavioral information as a novel model for the further study of welfare and production in the pasture-based dairy cow.

\section{MATERIALS AND METHODS}

\section{Study Area}

Data were collected at the Aberystwyth University dairy farm, Trawsgoed, Ceredigion, United Kingdom, between March and August of 2014. The farm was composed of approximately 200 Holstein and 150 JerseyFriesian crossbred cows managed in a semi-intensive all-year-round calving system. Cows on the farm were rotated and strip-grazed on leafy swards of perennial ryegrass (Lolium perenne) between March and October on a grazing platform of approximately 100 ha with an altitude of between 70 and $250 \mathrm{~m}$ above sea level. Cows were allocated approximately 2,500 kg of DM/ha of grass per area grazed leaving a grazing aftermath of approximately $1,500 \mathrm{~kg}$ of DM/ha. Cows in early lactation (0-120 DIM) were also buffer fed a TMR of grass [25 kg of total fresh weight $(\mathbf{F W})]$, maize silage $(10 \mathrm{~kg}$ of FW), rolled wheat ( $3 \mathrm{~kg}$ of FW), barley straw (0.3 $\mathrm{kg}$ of $\mathrm{FW}$ ), with the remainder consisting of a rumenprotected fat supplement, dairy mineral blend, and molasses during the evening. Early-lactation cows also received $3.60 \mathrm{~kg}$ of $\mathrm{FW}( \pm 0.81 \mathrm{~kg})$ of concentrates per day. Cows were milked twice daily at 0500 and $1600 \mathrm{~h}$.

\section{Grazing Management and GPS}

According to recent work (Kilgour, 2012), cows can exhibit up to 40 individual behaviors, though many of these are expressed in low abundance and for very short periods of time. Three main behaviors were identified and used in this work: grazing, resting, and walking. Hancock (1954) reported that the main behaviors of pasture-based dairy cattle were grazing and resting. Due to the reported difficulty in distinguishing between lying and standing by others using GPS data (Homburger et al., 2014), we decided that collating these 2 behaviors in equal proportion and representing them as resting would lead to less complex decision rules during the machine learning phase. We also decided to include walking as this is also frequently reported in behavioral studies (Robert et al., 2009; Silper et al., 2015). Grazing was identified when the cow's head was lowered and tearing at the pasture whether walking or standing still. Browsing (walking with her head close to the pasture) was also included within grazing. Resting was identified when the cow was lying or when she stood still with her head raised. Walking was identified when the cow was walking or running with her head raised. A total of 40 early-lactation (50-120 DIM) Holstein cows were used. Average parity and milk yield $(305 \mathrm{~d})$ of cows were $2.8 \pm 1.5$ lactations (mean $\pm \mathrm{SD})$ and $7,414 \pm 756 \mathrm{~kg}$ 
per cow, respectively. Cows selected had normal gait (Whay et al., 2003) and showed no other obvious signs of ill health. Cows were otherwise randomly selected for behavioral observation over the period of study and observed between the hours of 0800 and $1400 \mathrm{~h}$. This time period was chosen simply because it allowed for the longest period of observation between milking.

Four cows were observed on each day of observation from a distance of approximately $40 \mathrm{~m}$ by 2 observers from within a vehicle. This allowed clear visibility of the behavior exhibited by the observed cows and minimized the impact that human presence may have had on their behavior. Observation days varied depending on observer availability and weather conditions. For example, cows were not observed on days where adverse weather conditions were forecast such as heavy rain. This meant that the number of days of observation and observation days themselves varied from week to week. On average, approximately $3 \mathrm{~d}$ were between sampling days. Both observers had previously spent 2 unrecorded observational days refining observational techniques to maximize agreement in behavioral and transitional definition. This was to ensure that high-quality timestamped behaviors were recorded at and between each behavioral transition. Interobserver kappa coefficients for grazing, resting, and walking were 0.96, 0.99, and 1.00 , respectively, for the unrecorded behavioral days. During each observation period, cows were equipped with low-cost GlobalSat DG-100 Data Logger GPS receivers (GlobalSat Technology Corp., Taiwan). Receivers were mounted on collars around the neck and cows were strip-grazed in a separate paddock at the same stocking density of the remainder of the herd (average stocking density approximately: 16 cows/ha). Choosing to graze the observation cows in a separate field to the rest of the herd was to ensure that cows could be fully observed at all times, reducing the risk of losing sight of the observation cows among others in the herd. The pasture of the observation paddock was also Lolium perenne. The sward was measured during the entire data collection period so that each strip provided $2,500 \mathrm{~kg}$ of DM/ha of grass. Cows were managed so that the grazing residue was approximately $1,500 \mathrm{~kg}$ of DM/ ha. This management regimen was the same for the entire farm and meant that pasture quality changed very little during the experimental period. The aim was to replicate a well-managed grazing system as closely as possible. This routine was enough for a total daily grazing and observational period of $6 \mathrm{~h}$ for each experimental period. Four new cows were introduced into a fresh strip of grass each day with the same pasture allowance. Each observer was allocated 2 cows for each period of observation. The GPS receivers were chosen randomly from a collection that was rigorously field tested under the specific guidelines of the Institute of Navigation (ION, 1997). From an initial 36 receivers, 11 were discarded due to nonnormal positional fixes, leaving 25 for the study. The GPS receivers were programmed to sample the positional coordinates every $5 \mathrm{~s}$. From the calibration experiment (M. L. Williams, unpublished data), this was considered the optimal logging interval for both data collection and power consumption. Using a Casio F-91W time clock (Casio Electronics Co. Ltd., Shibuya, Tokyo, Japan) synchronized to the GPS receivers, behaviors were manually observed and recorded at each transition, to the second they occurred for the whole 6 -h observational period. Each 6 -h period yielded $24 \mathrm{~h}$ of high-quality time-stamped cow activity from the 4 cows observed. An independent test data set was used on the final classifier models. This consisted of $14 \mathrm{~h}$ of data gathered from 4 previously unobserved cows managed in a separate, undulating paddock, with these cows mixing with the rest of the herd. Pasture was not managed as stringently in this paddock, and it contained a sward of varying density. This was a purposeful choice and was made to test the stability and robustness of the final models.

\section{Data Sets}

Data were downloaded from each GPS receiver as a comma separated variable (csv) file and stored in a spreadsheet program. The GPS data sets for each cow contained the record number of each logged position, time (s), latitude, longitude, speed $(\mathrm{m} / \mathrm{s})$, and altitude $(\mathrm{m})$. The manually recorded field behaviors were matched alongside each row of GPS data. The compiled data set consisted of a total of $425 \mathrm{~h}$ ( $71 \mathrm{~h}$ of human observation) of behavioral and positional data. Behavioral sequences lasting at least $8 \mathrm{~min}$ were used for the analysis. This was done with the assumption that longer behavioral data sequences would provide a more reliable representation of the data sampled by the GPS units for each observed behavior. In total, $153 \mathrm{~h}$ of data was used for analysis: 28, 120, and $5 \mathrm{~h}$ for grazing, resting and walking, respectively.

\section{Data Preprocessing and Preparation}

Successive coordinates were exploited such that a variety of new features were extracted from the original data in 3 different phases. The approach detailed here is similar to that of earlier work (Godsk and Kjærgaard, 2011). However, the feature extraction and segmentation phases were modified. Briefly, the methodology employed is described below. 


\section{Phase 1}

Raw, ground truth behavior-labeled and timestamped GPS coordinates along with speed and altitude data were used to compute basic features for movement objects (MO). Contiguous data sequences for each behavioral class were selected from every data set represented by all 40 cows. An analysis of each consecutive GPS record allowed the calculation of the cow's distance traveled, speed, acceleration, absolute heading, and bearing from one data entry to the next, every $5 \mathrm{~s}$. Whether the cow was moving or not was determined using a simple adjustable Bayes induction filter. Three input parameters were used for adjustment of the filter: minimum speed (minimum speed required for determining movement), history length (the number of previous GPS records taken into account), and heading threshold (the heading threshold required for determining movement). Minimum speed and heading threshold were determined for all 25 GPS receivers using data collected from a separate dynamic test under guidelines set by the Institute of Navigation (ION, 1997). The average threshold values for all 25 GPS receivers were then calculated.

\section{Phase 2}

Data from phase 1 was grouped into segments of a predetermined size depending on the chosen segmentation strategy (i.e., the number of GPS instances to include when constructing each of the segments). Advanced features (AF) were then extracted for each segment based on the basic features from phase 1. In total, $43 \mathrm{AF}$ were extracted for phase 2 (Table 1). Of the behavioral classes under study, grazing and resting tended to be exhibited in the greatest proportion (Kilgour, 2012). Typically, dairy cattle graze in bouts of 7 min or less (Hejcmanová et al., 2009) with resting bouts lasting approximately $1 \mathrm{~h}$. However, for this work, we explored 3 different time intervals for segmentation to investigate whether the segmentation strategy would have any effect on classifier performance: 160,40 , and $10 \mathrm{~s}$. Thus, at a GPS sample rate of $5 \mathrm{~s}$, each segmentation interval comprised 32, 8, and $2 \mathrm{MO}$, respectively. Data for each segmentation strategy were evaluated separately. Once the data had been segmented at the desired interval, each group of segmented data then formed a segment object (SO). The SO contained the data for all $43 \mathrm{AF}$. Table 2 shows the unbalanced data sets for the 3 respective segmentation strategies with the corresponding number of SO.

We tested the data set where the decision classes (behaviors) were imbalanced and also balanced. The data instances were balanced by reducing the size of the behavioral category represented by the greatest number of SO (resting) to the same size as the category with the fewest SO. This is known as undersampling. We found that dairy cows exhibited very little walking behavior during the data collection phase and movement in itself was exhibited most of the time as grazing and cows spent the majority of their time grazing and resting. This could be due to the greater energy expenditure of walking thus this behavior may be limited especially in early-lactation cows (Dohme-Meier et al., 2014). Due to the great underrepresentation of walking in the data set, resting was reduced to the same level as grazing to preserve the integrity of the data set. Balancing the data instances was carried out using a subsampling method SpreadSubsample (Japkowicz and Stephen, 2002). This method produced a random undersample of the class with the greatest representation to a specified sample size. The size of the random undersampled class was predefined and was used to specify the number of SO for each individual data set. Table 2 shows the class distribution for the data set balanced using random undersampling.

\section{Phase 3}

The SO and their corresponding ground-truth behaviors were compiled and formatted such that they could be examined using the WEKA (Hall et al., 2009) data mining suite.

\section{Classifier Learning}

The popular data mining suite WEKA was used for the analysis of the data in this study. This suite of tools allows many different machine learning approaches to be used for comparative analysis and provides a variety of different metrics for assessing the performance of learners. The algorithms tested on the data used in this study were naïve Bayes (John and Langley, 1995), JRip (Cohen, 1995), J48 (Quinlan, 1993), and random forest (Breiman, 2001). Naïve Bayes is a simple technique for constructing classifiers, which are represented as vectors of feature values. The classifier considers each of the features to contribute independently (strong independence assumption) to the probability that an object belongs to a particular decision class. This is assumed regardless of any possible actual correlation(s) between features. The maximum likelihood is used as a metric to decide in which class a testing object belongs. J48 creates decision trees by choosing the most informative features and recursively partitioning the data into subtables based on their values. Each node in the tree represents a feature with branches from a node representing the alternative values this feature 
Table 1. Advanced features computed for each segment of GPS data to be used for behavioral classification

\begin{tabular}{|c|c|}
\hline Advanced feature & Movement type (unit) \\
\hline Accumulated time moving & Time (s) \\
\hline Accumulated time nonmoving & Time (s) \\
\hline Minimum speed & Speed $(\mathrm{m} / \mathrm{s})$ \\
\hline Mean speed & Speed $(\mathrm{m} / \mathrm{s})$ \\
\hline Maximum speed & Speed $(\mathrm{m} / \mathrm{s})$ \\
\hline Accumulated distance moving & Distance $(\mathrm{m})$ \\
\hline Accumulated distance nonmoving & Distance $(\mathrm{m})$ \\
\hline Maximum distance moving & Distance (m) \\
\hline Maximum distance nonmoving & Distance $(\mathrm{m})$ \\
\hline Movement percent left & Heading (\%) \\
\hline Movement percent right & Heading $(\%)$ \\
\hline Movement percent forward & Heading (\%) \\
\hline Movement percent U-turn & Heading (\%) \\
\hline Percent nonmoving & Heading (\%) \\
\hline Rate of change moving and nonmoving & (no.) \\
\hline Rate of change between any movement & (no.) \\
\hline Rate of change left & (no.) \\
\hline Rate of change right & (no.) \\
\hline Rate of change forward & (no.) \\
\hline Rate of change nonmoving & (no.) \\
\hline Rate of change U-turn & (no.) \\
\hline Minimum acceleration & Acceleration $\left(\mathrm{m} / \mathrm{s}^{2}\right)$ \\
\hline Maximum acceleration & Acceleration $\left(\mathrm{m} / \mathrm{s}^{2}\right)$ \\
\hline Mean acceleration positive & Acceleration $\left(\mathrm{m} / \mathrm{s}^{2}\right)$ \\
\hline Mean acceleration negative & Acceleration $\left(\mathrm{m} / \mathrm{s}^{2}\right)$ \\
\hline Accumulated acceleration positive & Acceleration $\left(\mathrm{m} / \mathrm{s}^{2}\right)$ \\
\hline Accumulated acceleration negative & Acceleration $\left(\mathrm{m} / \mathrm{s}^{2}\right)$ \\
\hline Changes between positive and negative acceleration & (no.) \\
\hline Heading accumulated left & (no.) \\
\hline Heading accumulated right & (no.) \\
\hline Heading accumulated forward & (no.) \\
\hline Heading accumulated nonmoving & (no.) \\
\hline Heading accumulated U-turn & (no.) \\
\hline Maximum heading change left & Heading $\left({ }^{\circ}\right)$ \\
\hline Maximum heading change right & Heading $\left({ }^{\circ}\right)$ \\
\hline Maximum heading change forward & Heading $\left({ }^{\circ}\right)$ \\
\hline Maximum heading change nonmoving & Heading $\left(^{\circ}\right)$ \\
\hline Maximum heading change U-turn & Heading $\left(^{\circ}\right)$ \\
\hline Mean heading change per left & Heading $\left({ }^{\circ}\right)$ \\
\hline Mean heading change per right & Heading $\left({ }^{\circ}\right)$ \\
\hline Mean heading change per forward & Heading $\left(^{\circ}\right)$ \\
\hline Mean heading change per nonmoving & Heading $\left({ }^{\circ}\right)$ \\
\hline Mean heading change per U-turn & Heading $\left(^{\circ}\right)$ \\
\hline
\end{tabular}

can take according to the current subtable. Partitioning stops when all data items in the subtable have the same classification. A leaf node is then created, and this classification is assigned. JRip learns propositional rules by repeatedly growing rules and pruning them. During the growth phase, antecedents are added greed-

Table 2. Number of segment objects created for each behavior in unbalanced data sets and data sets balanced by random undersampling

\begin{tabular}{|c|c|c|c|c|c|c|}
\hline \multirow{2}{*}{$\begin{array}{l}\text { Segmentation strategy }{ }^{1} \\
\text { (movement object/segment) }\end{array}$} & \multicolumn{6}{|c|}{ Segment objects ${ }^{2}$} \\
\hline & \multicolumn{3}{|c|}{ Unbalanced data sets } & \multicolumn{3}{|c|}{ Balanced data sets } \\
\hline 32 & 631 & 2,703 & 96 & 631 & 631 & 96 \\
\hline 8 & 1,864 & 5,238 & 407 & 1,864 & 1,864 & 407 \\
\hline 2 & 9,921 & 45,069 & 1,612 & 9,921 & 9,921 & 1,612 \\
\hline
\end{tabular}

${ }^{1}$ Movement objects present per segment. For example, 32 movements equates to $160 \mathrm{~s}$ of GPS data (at a 5-s GPS sample rate) per segment object.

${ }^{2}$ Each segment object contained data for all advanced features for the expressed behavior. 
ily until a termination condition is satisfied. Antecedents are then pruned in the next phase subject to a pruning metric. Once the ruleset is generated, a further optimization is performed where rules are evaluated and deleted based on their performance on randomized data. Random forest is an ensemble learning method for classification. It works by constructing a collection ("forest") of (random) decision trees at training time and returning the class that is the mode of all of the classes of the individual trees. Random forest classifiers attempt to mitigate the tendency of decision trees to overfit the training data set. The reasoning behind these particular choices was to try to provide a realistic set of results and also to show the different characteristics of the learners themselves. Naïve Bayes is a pessimistic learner and therefore biased but stable (Bouckaert, 2008). J48 is an optimistic learner and it is therefore unbiased, suffers from high variance, and is thus unstable (Bouckaert, 2008). JRip is a rule-based classifier and lies somewhere in between Naïve Bayes and J48. Random forest attempts to reduce variance (and therefore total error).

\section{Classifier Validation Strategy}

Classifier performance was evaluated using stratified 10-fold cross-validation (10-FCV). In 10-FCV, the data set is randomly split into 10 folds or subsets of data where class representation is preserved in the same proportion (as far as possible) as the full data set. The first 9 folds are then used to learn a classifier, whereas the tenth is used for validation. This process is then repeated each time using a single fold for validation with the remainder used for training. This continues until all 10 folds have been used for both training and validation resulting in an average classification accuracy (CA) and error rate. For the results generated here, 10-FCV was repeated for 10 different randomizations/ runs $(10 \times 10$-FCV $)$ of the data. This helped to learn more robust classifiers and was done for all data sets in Table 2. Finally, the best performing classifier models were applied to a previously unseen independent test data set.

\section{Selection of Advanced Features}

In addition, the redundancy and relevance of the features extracted from the GPS data (AF) were investigated for the best performing classifiers to explore which features were most useful in classifying the 3 behaviors. Advanced features were evaluated using CFS (Hall, 1998), which selects subsets of features that are highly correlated with the class value and that have low correlation with each other. A "greedy hill-climbing" approach was used to perform the search through the space of AF. Classifier performance and stability was then evaluated using the best performing data set in Table 2 with 10-FCV.

\section{Performance Evaluation}

The overall CA was one of the metrics used to assess performance. This is the most commonly reported and intuitive metric for classifier performance. It serves as a general indicator of the efficiency of a model to correctly predict all of the behavioral classes:

$$
\text { Classification accuracy }=\frac{\mathrm{TP}+\mathrm{TN}}{\mathrm{TP}+\mathrm{FP}+\mathrm{FN}+\mathrm{TN}},
$$

where TP is the number of true positives, $\mathrm{TN}$ is the number of true negatives, FP is the number of false positives, and FN is the number of false negatives. However, as a stand-alone metric of performance of multi-class problems, CA does not compensate for success that is due to mere chance.

$$
\text { False positive rate }(\mathbf{F P R})=\frac{\mathrm{FP}}{\mathrm{FP}+\mathrm{TN}},
$$

which is the proportion of instances incorrectly labeled as positive instances.

$$
\text { F-measure }=\frac{2 \times \text { Precision } \times \text { Recall }}{\text { Precision }+ \text { Recall }}
$$

where precision $=\mathrm{TP} /(\mathrm{TP}+\mathrm{FP})$, and recall $=\mathrm{TP} /$ $(\mathrm{TP}+\mathrm{FN})$. The F-measure is defined as the harmonic mean of precision and recall.

Area under the receiver operating curve (AUC) was represented as

$$
\mathrm{AUC}=(\mathrm{TP}+\mathrm{TN}) / 2,
$$

which is a measure of the discriminatory power of a classifier which measures the area under the receiver operating characteristic (ROC) curve (Fawcett, 2006). The ROC curve is constructed using the total true positives (y-axis) and the total true negatives (x-axis). A classifier generating more true positives and fewer false positives is preferable to the opposite. It should be noted that AUC is not without its problems when used as a metric in and of itself for the classification problem (Hand, 2009) and this is the reason for including several other performance metrics above.

All statistical analysis was undertaken using GenStat Fourteenth Edition (VSN International, Hemel Hemp- 
Table 3. Weighted classification accuracy of all classifier learners on unbalanced data for grazing, resting, and walking for 32-, 8-, and 2-segment object strategies using 10-fold cross validation

\begin{tabular}{lccccc}
\hline & & \multicolumn{3}{c}{ Classification accuracy } \\
\cline { 2 - 5 } Classifier & $\begin{array}{c}\text { Segmentation } \\
\text { strategy }^{1}\end{array}$ & Grazing & Resting & Walking & $\begin{array}{c}\text { Weighted } \\
\text { average }\end{array}$ \\
\cline { 3 - 5 } Näive Bayes & 32 & 0.72 & 0.71 & 0.99 & 0.72 \\
& 8 & 0.71 & 0.64 & 0.99 & 0.68 \\
JRip & 2 & 0.72 & 0.60 & 1.00 & 0.64 \\
& 32 & 0.56 & 0.92 & 0.98 & 0.85 \\
J48 & 8 & 0.48 & 0.89 & 0.98 & 0.79 \\
& 2 & 0.09 & 0.98 & 0.96 & 0.82 \\
Random forest & 32 & 0.56 & 0.90 & 0.98 & 0.84 \\
& 8 & 0.50 & 0.86 & 0.98 & 0.87 \\
& 2 & 0.16 & 0.95 & 0.95 & 0.86 \\
& 32 & 0.53 & 0.93 & 0.99 & 0.79 \\
& 8 & 0.51 & 0.88 & 0.99 & 0.81 \\
\hline
\end{tabular}

${ }^{1}$ Segmentation strategy was the number of movements [or global positioning system (GPS) instances] per data segment. For example, the 32-segment object segmentation strategy equates to $160 \mathrm{~s}$ of GPS data (at a 5-s GPS sample rate) per segment object.

stead, UK; footnotes below tables give information on the statistical tests used).

\section{RESULTS}

Classification accuracy was first compared with the unbalanced data sets (Table 2). Weighted average CA across all segmentation strategies was best for JRip and random forest (Table 3). Class imbalance can lead to the reinforcement of the majority class in the learned rules, thus leading to domination by a particular class (Kohavi, 1995). In this case, resting represented on average $76 \%$ of the SO in each data set and had high CA (Table 3). Interestingly, walking, which represented the behavioral class with fewest instances (Table 2), had the highest comparable CA (Table 3); this was due to the easily distinguishable characteristics obtained for this class. Due to the relative underrepresentation of grazing in each data set and the apparent confusion between this class and resting, class-specific CA was generally poor for this behavior particularly for the 2 SO data set. To address this, the data set was balanced by random undersampling (Table 2). The success of each classifier was determined based on relative performance for each segmentation strategy. The best classifiers were JRip and random forest when measured across all metrics regardless of the segmentation strategy employed (Table 4). The effect of balancing the class representation for the individual behaviors was investigated using only JRip and random forest because of their superior performance (Figure 1) when compared with naïve Bayes and J48. Additionally, performance for all metrics suffered as the data set was segmented into smaller sized $\mathrm{SO}$ and best performance was found when SO were composed of $32 \mathrm{MO}$ (Table 4). This effect on performance is linked to the averaging effects of the inclusion of greater numbers of SO. Hence, larger segments produce more easily definable MO.

Table 4. Weighted average classifier learner performance on data balanced by random undersampling under 32-, 8-, and 2-segment object strategy using 10 -fold cross validation

\begin{tabular}{|c|c|c|c|c|c|c|c|c|c|c|c|c|c|}
\hline Metric $^{1}$ & \multicolumn{3}{|c|}{ Naive Bayes } & \multicolumn{3}{|c|}{ JRip } & \multicolumn{3}{|c|}{ J48 } & \multicolumn{3}{|c|}{ Random forest } & SEM \\
\hline CA & $0.73^{\mathrm{c}}$ & $0.71^{\mathrm{c}}$ & $0.69^{c}$ & $0.81^{\mathrm{a}}$ & $0.76^{\mathrm{a}}$ & $0.73^{\mathrm{a}}$ & $0.78^{\mathrm{b}}$ & $0.73^{\mathrm{b}}$ & $0.72^{\mathrm{b}}$ & $0.81^{\mathrm{a}}$ & $0.76^{\mathrm{a}}$ & $0.72^{\mathrm{b}}$ & 0.002 \\
\hline FPR & $0.23^{\mathrm{a}}$ & $0.22^{\mathrm{a}}$ & $0.23^{\mathrm{b}}$ & $0.17^{\mathrm{c}}$ & $0.20^{\mathrm{b}}$ & $0.23^{\mathrm{b}}$ & $0.19^{\mathrm{b}}$ & $0.22^{\mathrm{a}}$ & $0.24^{\mathrm{a}}$ & $0.16^{\mathrm{c}}$ & $0.20^{\mathrm{b}}$ & $0.24^{\mathrm{a}}$ & 0.002 \\
\hline F-measure & $0.72^{\mathrm{c}}$ & $0.70^{\mathrm{c}}$ & $0.69^{c}$ & $0.81^{\mathrm{a}}$ & $0.76^{\mathrm{a}}$ & $0.73^{\mathrm{a}}$ & $0.78^{\mathrm{b}}$ & $0.73^{\mathrm{b}}$ & $0.72^{\mathrm{b}}$ & $0.81^{\mathrm{a}}$ & $0.76^{\mathrm{a}}$ & $0.72^{\mathrm{b}}$ & 0.002 \\
\hline AUC & $0.82^{\mathrm{c}}$ & $0.82^{\mathrm{b}}$ & $0.79^{\mathrm{c}}$ & $0.85^{\mathrm{b}}$ & $0.83^{\mathrm{b}}$ & $0.80^{\mathrm{b}}$ & $0.81^{\mathrm{d}}$ & $0.76^{\mathrm{c}}$ & $0.78^{\mathrm{d}}$ & $0.90^{\mathrm{a}}$ & $0.86^{\mathrm{a}}$ & $0.81^{\mathrm{a}}$ & 0.002 \\
\hline
\end{tabular}

${ }^{\mathrm{a}-\mathrm{d}}$ Means within a row with different superscripts are compared between classifiers for each corresponding segmentation strategy and differ $(P$ $<0.05)$.

${ }^{1}$ Means were tested using ANOVA and Tukey's posthoc test. $\mathrm{CA}=$ classification accuracy; FPR $=$ false positive rate; AUC $=$ area under the receiver operating curve.

${ }^{2}$ Segmentation strategy was the number of movements (or GPS instances) per data segment. For example, the 32-segment object segmentation strategy equates to $160 \mathrm{~s}$ of GPS data (at a 5-s GPS sample rate) per segment object. 


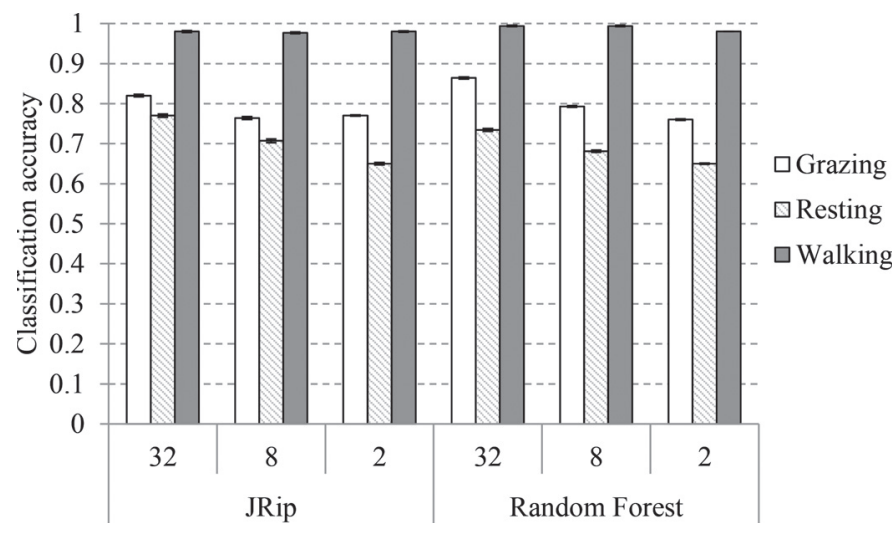

Figure 1. Average classification accuracy $( \pm$ SE) of JRip and random forest classifier learners across all 3 behaviors according to segmentation strategy and data balanced by random undersampling.

Balancing the data sets by random undersampling led to an improvement in CA for grazing relative to the previous performance $(\mathrm{JRip}=+0.26,+0.28$, and +0.68 ; random forest $=+0.33,+0.28$, and +0.52 for 32,8 , and $2 \mathrm{SO}$, respectively) but a reduction in performance for resting (JRip $=-0.15,-0.18$, and -0.33 ; random forest $=-0.20,-0.20$, and -0.28 for 32,8 , and $2 \mathrm{SO}$, respectively). The effect of class balancing the data on walking was negligible. It is clear that these 2 algorithms have a greater ability to classify behaviors where physical movement is a prominent feature of the data. Resting behavior was sometimes confused with grazing for all learners, probably because of the inherent error of the GPS device and therefore the perceived subsequent movement of cows between consecutive fixes (Lewis et al., 2007), when in fact they are resting.

Due to the superior performance of JRip and random forest when compared with other learners (Table 4 ), further validation and testing took place with these learners and the 32 SO data set only. During observation, cows generally exhibited each behavior for periods exceeding $3 \mathrm{~min}$; therefore, the $32 \mathrm{SO}$ strategy coincided well with average behavioral duration. An independent test set consisting of $14 \mathrm{~h}$ of ground truth behavior-labeled and time-stamped GPS coordinates was used for final model testing that was segmented at $32 \mathrm{SO}$. The final model as derived from the validation phase for JRip is shown (Table 5) along with its respective success rates during modeling. It was interesting to note the induced rules and their antecedent values for each of the behaviors. Many of these were informative, and because JRip generates rules that are transparent to human scrutiny, it offered an insight into how features of physiological movement of cows helped to characterize the behavior. For example, it could be seen that walking was so easy to classify that only a single antecedent was required to predict the behavior: maximum speed. This was both informative and realistic because if a healthy cow was walking in a given direction it would always exceed this threshold $(\geq 1.10 \mathrm{~m} / \mathrm{s})$. The rules also helped to reflect the complex nature of grazing behavior due to the rule clarity and number of actual rules. This complexity should not be interpreted as a poor reflection of the learner, but rather that grazing, because of the physical nature, involves elements of both walking and resting because the cow may stop for a short period while it tears at pasture before moving on to the next area. Thus, many different antecedents were involved in building a model to represent it. No significant differences were found in any of the performance measures (Table 6) for JRip and random forest on the independent test set. These results were encouraging because data for the test set were purposely retrieved from cattle grazing an undulating, densely stocked paddock where pasture conditions were not controlled as stringently as the area where the data were gathered for model building and validation. Classification accuracy of individual behaviors (Figure 2) was more balanced across the behaviors for JRip when compared with random forest. Walking behavior, in both examples, was classified with almost $100 \%$ accuracy.

A feature selection and stability assessment was performed on the original $32 \mathrm{SO}$ training data. Table 7 gives a comparison of the performance of JRip and random forest both with and without $\mathrm{AF}$ selection as tested by 10-FCV. No significant improvement or degradation was found in classifier performance when the AF with the highest contributions during modeling of the observed behaviors were used (Table 7). Figure 3 illustrates the average number of folds in which the most important AF appeared during 10-FCV (10 runs $\times 10$ folds). Among the 13 selected AF (Figure 3), features 2, 5,6 , and 7 (i.e., maximum heading change nonmoving, minimum speed, mean speed, and maximum speed) were selected in every fold. This indicated that it may not be necessary to consider extracting all of the $\mathrm{AF}$ listed in Table 1 for future work in this field. Although a marginal improvement was found in performance for random forest, this was nonsignificant. Furthermore, both classifiers had a small standard deviation and therefore good stability for all measures of performance. However, despite the comparable performance of JRip and random forest, the simplicity of the rule set generated by JRip (Table 5) is advantageous because it provides a model that is transparent and humanly interpretable through the use of intuitive if-then rules. 


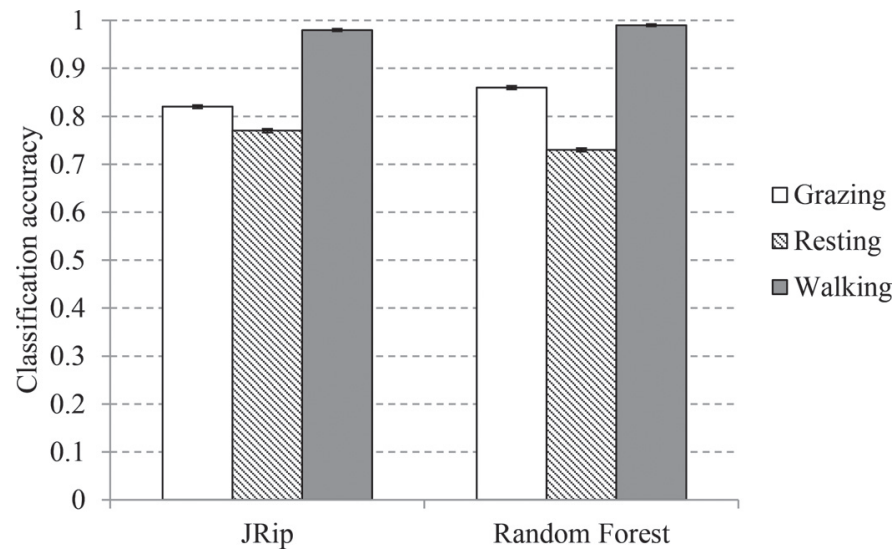

Figure 2. Average classification accuracy $( \pm \mathrm{SE})$ of final models of JRip and random forest classifier learners across all 3 behaviors on an independent, balanced 32-segment object test set.

\section{DISCUSSION}

The objective of this work was to build a robust model of the most notable behaviors of the pasturebased dairy cow: grazing, resting, and walking and to fully document how this was derived. We have achieved greater performance than other comparable studies using GPS only to classify the most commonly represented behavioral activities (Homburger et al., 2014). Homburger et al. (2014) used a logging interval of 20 $\mathrm{s}$ to record positional fixes achieving an overall $\mathrm{CA}$ of $77 \%$. Longer fix intervals can create uncertainty about the location and the subsequent intra-sample behavior exhibited by cattle (Swain et al., 2008). This can lead to inaccuracies in distance traveled and speed between fixes (Pépin et al., 2004). Speed and distance metrics featured heavily in the final model generated using the JRip learner for grazing and walking behaviors in this work, and indeed this was reflected in the learned rules.

Cattle could of course be grazing or walking in several different directions in the period between samples, limiting the use of the extracted features to distinguish behaviors. The final models in this study were based on 40 cows and $153 \mathrm{~h}$ of time-stamped cow behavior gathered over a period of 4 mo. To our knowledge, this is the most extensive data set used for the preparation of such models. We believe this was sufficient to account for conditions out of our experimental control such as atmospheric effects and dilution of GPS precision (ION, 1997). Furthermore, the models achieved high performance when tested on an independent test set where cows were grazed outside experimentally controlled conditions. Unaccounted for in the training data were multipath effects, errors in GPS output created by topographical features and buildings, for example (Cai et al., 2014). However, the independent test set contained instances where cows were standing next to trees, hedgerows, and buildings, suggesting that overall performance in the test set may have been higher had cows been restricted to areas where GPS signal-to-noise ratio was lower. We deem the behaviors under study not only to be the most easily definable when using temporal movement metrics produced from GPS receivers, but perhaps also the most important for monitoring cow health in pasture-based cows. Lying times have been shown to increase in both severely lame and clinically lame cows (Sepúlveda-Varas et al., 2014), and standing time has been shown to be higher in cows with ketosis (Itle et al., 2015). It seems that behavior is also affected by age, parity, and disease. For

Table 5. Rule set generated by the JRip classifier learner for classifying dairy cow behavior using advanced features created from GPS data

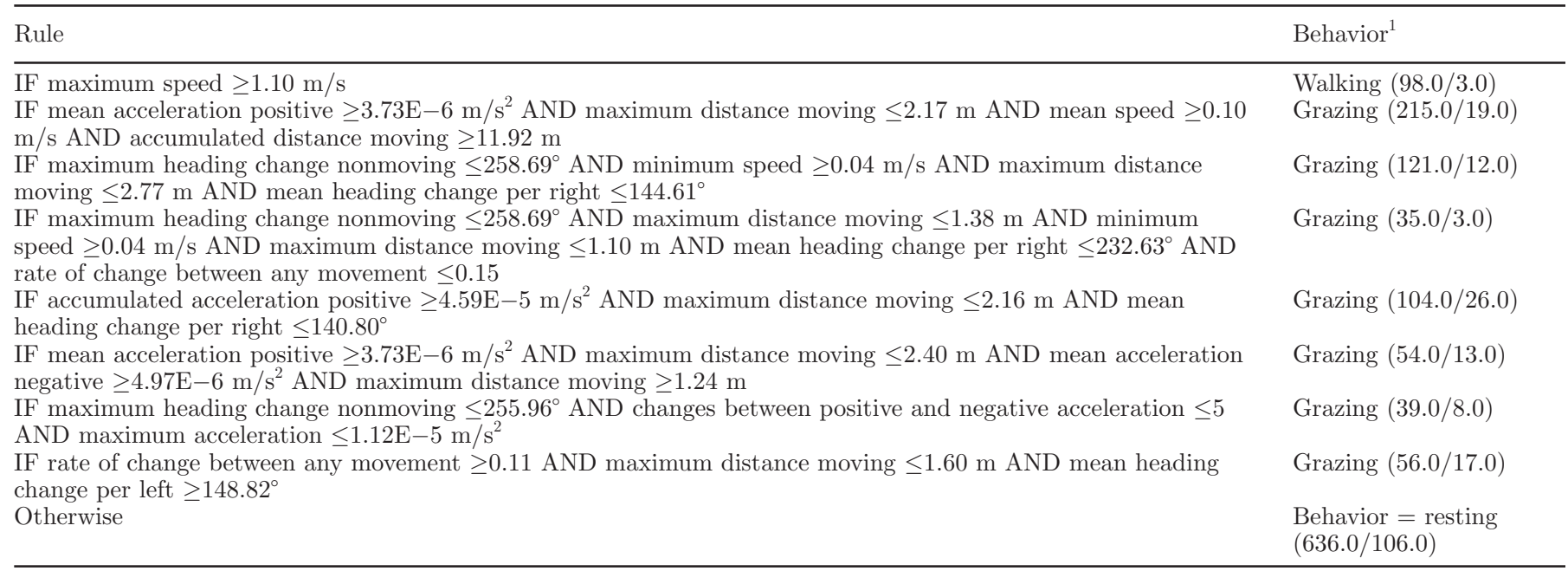

\footnotetext{
${ }^{1}$ Numbers in parentheses below behavioral classes indicate number of successful classifications/errors in the balanced training data.
} 
Table 6. Average performance of final models of JRip and random forest classifier learners on an independent, balanced 32-segment object test set

\begin{tabular}{lcccc}
\hline Metric $^{1}$ & JRip & Random forest & SEM & $P$-value \\
\hline CA & 0.85 & 0.83 & 0.008 & $\mathrm{NS}^{2}$ \\
FPR & 0.10 & 0.17 & 0.010 & $\mathrm{NS}$ \\
F-Measure & 0.76 & 0.77 & 0.013 & $\mathrm{NS}$ \\
AUC & 0.87 & 0.92 & 0.009 & $\mathrm{NS}$ \\
\hline
\end{tabular}

${ }^{1}$ Tested using 2 sample $t$-test. $\mathrm{CA}=$ classification accuracy; $\mathrm{FPR}=$ false positive rate; $\mathrm{AUC}=$ area under the receiver operating curve.

${ }^{2} \mathrm{NS}=P>0.05$.

example, Charlton et al. (2015) found that cows with hock and knee injuries spent less time lying compared with cows without injuries and Steensels et al. (2012) found that lying times increased significantly with age in housed cows. To our knowledge, little work exists in the long-term monitoring of behavioral changes associated with disease in pasture-based dairy cows and is therefore the long-term objective of the current work. Information has already been gathered on the effect of disease on the feeding behavior of housed cows (Huzzey et al., 2007; González et al., 2008) and therefore more is needed for long-term assessment of behavior in cows at pasture. Inter-cow comparisons are likely to be of lower value than long-term intra-cow behavior for disease diagnostics (González et al., 2008; Reader et al., 2011).

As well as the most notable behaviors, other authors (Dutta et al., 2015) have had success in classifying other, less frequently exhibited behaviors, for example, rubbing, scratching, and licking. These behaviors are often combined into a single class due to their relatively poor representation in general bovine behavior. Recent success has been achieved in classifying data from other cow-mounted sensors. For example, Nielsen (2013) successfully classified cattle grazing (sensitivity $=83.63 \%$; specificity $=90.20 \%$; precision $=85.75 \%$ ) when data from a 3D head-mounted activity sensor were coupled with data from a 3-dimensional leg-mounted accelerometer. Robert et al. (2009) classified lying and standing behaviors of 15 calves to a very high accuracy $(\mathrm{CA}=99$ and $98 \%$, respectively) using accelerometers however, less success was achieved in classifying walking (CA $=68 \%)$. We achieved high CA with JRip and random forest on the independent test set (JRip $=0.85$; random forest $=0.83)$. Both JRip and random forest also achieved a false discovery rate $(0.10$ versus 0.17 , respectively) comparable with other published work (Dutta et al., 2015). In comparison with such work, Pluk et al. (2012) and Viazzi et al. (2014) describe methods of automatically identifying changes in the posture of dairy cows using video recordings to automatically detect lameness with some success. Whereas this type of work sets a strong benchmark for making gait classifications more reliable, it is less time consuming and reduces the subjectivity associated with manual gait assessment, and does little to detect the early onset of lameness. Cows are often described as stoic prey animals and may not display obvious gait abnormalities during the early onset of a lameness problem for example. This may account for the little change that is seen between the lower end of mobility scoring systems and it may only be when the pain has become unbearable that cows eventually show signs of an altered gait. As shown by Reader et al. (2011), decreases in milk yield were evident $7 \mathrm{wk}$ before the manifestation of the later diagnosed lameness.

Due to its simplicity, CA is the most commonly used evaluation metric for model performance. However, it does not compensate for results that are due to chance (Ben-David, 2008). The strength of a model can also be defined by the area under the receiver operating charac-

Table 7. Weighted average performance and stability $( \pm \mathrm{SD})$ of JRip and random forest classifier learners before and after selection of advanced features on original 32-segment object data set balanced by random undersampling using 10 -fold cross validation

\begin{tabular}{|c|c|c|c|c|c|c|c|c|}
\hline Metric $^{1}$ & \multicolumn{4}{|c|}{ JRip } & \multicolumn{4}{|c|}{ Random forest } \\
\hline FPR & $0.17(0.03)$ & $0.16(0.03)$ & 0.003 & NS & $0.16(0.03)$ & $0.15(0.03)$ & 0.003 & NS \\
\hline F-measure & $0.81(0.04)$ & $0.81(0.04)$ & 0.004 & NS & $0.81(0.03)$ & $0.82(0.03)$ & 0.003 & NS \\
\hline AUC & $0.85(0.03)$ & $0.85(0.03)$ & 0.003 & NS & $0.90(0.03)$ & $0.91(0.03)$ & 0.003 & NS \\
\hline
\end{tabular}

${ }^{1}$ Tested using 2-sample $t$-test. $\mathrm{CA}=$ classification accuracy; FPR $=$ false positive rate; AUC = area under the receiver operating curve.

${ }^{2} \mathrm{NS}=(P>0.05)$. 


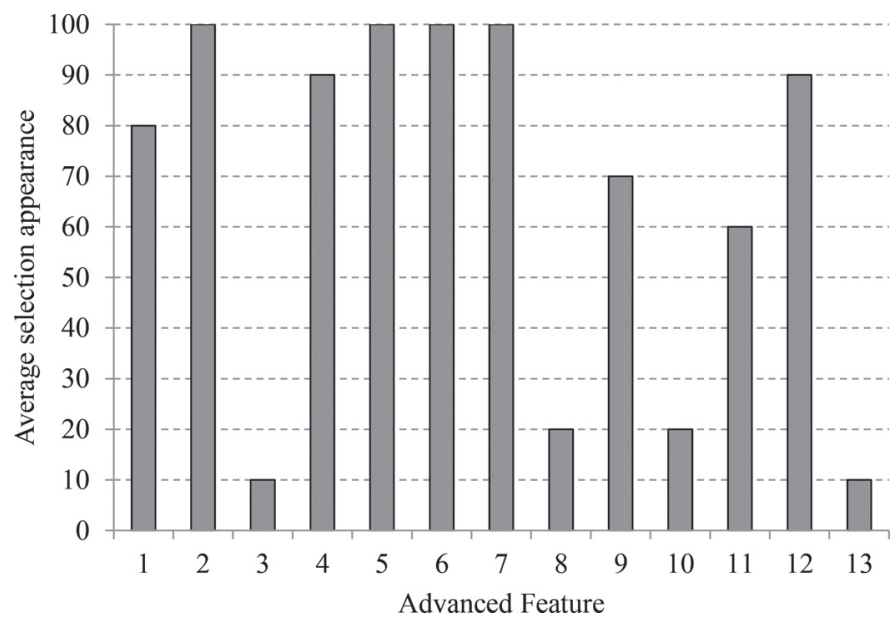

Figure 3. Average selection appearances of the most valuable advanced features. The advanced features from 1 to 13 correspond to (1) movement percent forward, (2) maximum heading change nonmoving, (3) mean heading change percent left, (4) mean heading change percent right, (5) minimum speed, (6) mean speed, (7) maximum speed, (8) mean acceleration positive, (9) mean acceleration negative, (10) accumulated acceleration positive, (11) accumulated acceleration negative, (12) accumulated distance moving, (13) maximum distance moving.

teristic (ROC) curve, known as the AUC. Graphically, in the ROC space the diagonal line $\mathrm{y}=\mathrm{x}$ represents a classification strategy that randomly guesses a class (Fawcett, 2006). Larger AUC values are preferred and represent a ROC curve that is positioned toward the top left corner of the ROC space. JRip and random forest performed very well on the independent test set (0.87 versus 0.92 , respectively).

Despite the similar performance of JRip and random forest, the simplicity of JRip and comparable execution time makes it the favored choice of the 2 for this particular work. JRip uses a global optimization phase on an initial set of rules built from scratch, and consequently, the resulting model has reduced complexity. Previous studies (Swain et al., 2008; Forin-Wiart et al., 2015) found that increasing the GPS fix rate provided a more accurate account of the movement of animals. Fix rate did not have a significant effect on absolute accuracy during static accuracy tests (M. L. Williams, unpublished data); therefore, the only limitation to data collection was battery power. We found that the optimal fix rate for maximum data acquisition and power consumption for the GlobalSat DG-100 GPS receiver was $5 \mathrm{~s}$ (M. L. Williams, unpublished data). This allowed for approximately 17,000 fixes to be recorded in $24 \mathrm{~h}$ on each receiver, equating to approximately $28 \%$ of the capacity of the receiver. We have already measured the stationary accuracy of these receivers in conditions where for example the receiver may come into close proximity with hedgerows. For the applica- tion of our model in the further study of dairy cow behavior in uncontrolled conditions, these factors will be taken into account.

Practically, the simplicity of placing each receiver onto cows was as simple as placing any other management tool on cow collars, for example, a pedometer for measuring activity in reproductive management. With minimal positional adjustment required, the use of small GPS receivers may be useful in a commercial environment. We believe that the versatility and the high level of performance attained in the present work constitute a strong case for the use of small, discrete, low cost (approximately $\$ 60$ US) GPS receivers mounted on neck collars. Using a variety of features to describe the behavior of cattle from GPS data, we have produced a humanly interpretable model of the main behaviors that can be used for further study. This model extends on the success of others in identifying cattle behavior (de Weerd et al., 2015; Dutta et al., 2015) and uses movement features that can be further exploited in understanding the behavior of cows at pasture from GPS only. Nevertheless, power consumption is a weakness of current GPS receivers and more work on this aspect is required in their development for practical application. As well as behavioral identification, the flexibility of GPS would also allow herdsmen to retrieve positional fixes as well as data on distances traveled by cows and their energy expenditure. This could assist in making more informed decisions on future nutritional management as well as the possibility of monitoring health status over time.

\section{CONCLUSIONS}

The results of this work illustrate the application of data mining techniques to features extracted from temporal GPS data. Furthermore, it highlights the wealth of data that can be gathered from small, low-cost GPS receivers alone. The success rate of our final model using JRip highlights its ability to classify the main behaviors exhibited by pasture-based dairy cows in a way that is transparent to human scrutiny. The next step will be to identify GPS data sequences representative of behavioral transitions. This model can then be used to track temporal changes in the behavior of pasture-based dairy cows afflicted by disease to work toward providing a more objective means of early disease identification.

\section{ACKNOWLEDGMENTS}

The authors thank the farm staff at Aberystwyth University Research Farm (Abeyrstwyth, Cerediginon, UK) for their cooperation. The sponsorship of M. Wil- 
liams by Coleg Cymraeg Cenedlaethol (Carmarthen, Wales, UK) is gratefully acknowledged.

\section{REFERENCES}

Ben-David, A. 2008. About the relationship between ROC curves and Cohen's kappa. Eng. Appl. Artif. Intell. 21:874-882. http://dx.doi. org/10.1016/j.engappai.2007.09.009.

Bicalho, R. C., S. H. Cheong, G. Cramer, and C. L. Guard. 2007. Association between a visual and an automated locomotion score in lactating Holstein cows. J. Dairy Sci. 90:3294-3300. http://dx.doi. org/10.3168/jds.2007-0076.

Bouckaert, R. R. 2008. Practical bias variance decomposition. Pages 247-257 in AI 2008: Adv. in Artif. Intell. Springer Berlin Heidelberg, Germany.

Breiman, L. 2001. Random forests. Mach. Learn. 45:5-32.

Cai, C., Y. Gao, L. Pan, and W. Dai. 2014. An analysis on combined GPS/COMPASS data quality and its effect on single point positioning accuracy under different observing conditions. Adv. Space Res. 54:818-829. http://dx.doi.org/10.1016/j.asr.2013.02.019.

Charlton, G. L., V. Bouffard, J. Gibbons, E. Vasseur, D. B. Haley, D. Pellerin, and A. M. de Passillé. 2015. Can automated measures of lying time help assess lameness and leg lesions on tie-stall dairy farms? Appl. Anim. Behav. Sci. http://dx.doi.org/10.1016/j. applanim.2015.02.011.

Cohen, W. W. 1995. Fast effective rule induction. Pages $115-123$ in Twelfth Int. Conf. on Machine Learning. Morgan Kaufmann, Burlington, MA.

de Weerd, N., F. van Langevelde, H. van Oeveren, B. A. Nolet, A Kölzsch, H. H. Prins, and W. F. de Boer. 2015. Deriving animal behavior from high-frequency GPS: Tracking cows in open and forested habitat. PLoS ONE http://dx.doi.org/10.1371/journal. pone.0129030.

Dohme-Meier, F., L. D. Kaufmann, S. Görs, P. Junghans, C. C. Metges, H. H. van Dorland, and A. Münger. 2014. Comparison of energy expenditure, eating pattern and physical activity of grazing and zero-grazing dairy cows at different time points during lactation. Livest. Sci. 162:86-96. http://dx.doi.org/10.1016/j. livsci.2014.01.006.

Dutta, R., D. Smith, R. Rawnsley, G. Bishop-Hurley, J. Hills, G. Timms, and D. Henry. 2015. Dynamic cattle behavioral classification using supervised ensemble classifiers. Comput. Elect. Agric. 111:18-28. http://dx.doi.org/10.1016/j.compag.2014.12.002.

Dyer, R. M., N. K. Neerchal, U. Tasch, Y. Wu, P. Dyer, and P. G. Rajkondawar. 2007. Objective determination of claw pain and its relationship to limb locomotion score in dairy cattle. J. Dairy Sci. 90:4592-4602. http://dx.doi.org/10.3168/jds.2007-0006.

Fawcett, T. 2006. An introduction to ROC analysis. Pattern Recognit. Lett. 27:861-874. http://dx.doi.org/10.1016/j.patrec.2005.10.010.

Forin-Wiart, M. A., P. Hubert, P. Sirguey, and M. L. Poulle. 2015. Performance and accuracy of lightweight and low-cost GPS data loggers according to antenna positions, fix intervals, habitats and animal movements. PLoS ONE 10:e129271. http://dx.doi. org/10.1371/journal.pone.0129271.

Godsk, T., and M. B. Kjærgaard. 2011. High classification rates for continuous cow activity recognition using low-cost GPS positioning sensors and standard machine learning techniques. Pages 174 188 in Adv. Data Mining. Applications and Theoretical Aspects:. Springer Berlin Heidelberg, Germany.

González, L. A., B. J. Tolkamp, M. P. Coffey, A. Ferret, and I. Kyriazakis. 2008. Changes in feeding behavior as possible indicators for the automatic monitoring of health disorders in dairy cows. J. Dairy Sci. 91:1017-1028. http://dx.doi.org/10.3168/jds.2007-0530.

Hall, M., E. Frank, G. Holmes, B. Pfahringer, P. Reutemann, and I. H. Witten. 2009. The WEKA data mining software: an update. SIGKDD Explor. 11:10-18. http://dx.doi. org/10.1145/1656274.1656278.

Hall, M. A. 1998. Correlation-Based Feature Subset Selection for Machine Learning. PhD thesis, Department of Computer Science, University of Waikato, Hamilton, New Zealand.
Hancock, J. 1954. Studies of grazing behavior in relation to grassland management I. Variations in grazing habits of dairy cattle. J. Agric. Sci. 44:420-433. http://dx.doi.org/10.1017/S0021859600045287.

Hand, D. J. 2009. Measuring classifier performance: A coherent alternative to the area under the ROC curve. Mach. Learn. 77:103-123.

Hejcmanová, P., M. Stejskalová, V. Pavlů, and M. Hejcman. 2009 Behavioral patterns of heifers under intensive and extensive continuous grazing on species-rich pasture in the Czech Republic. Appl. Anim. Behav. Sci. 117:137-143. http://dx.doi.org/10.1016/j. applanim.2009.01.003.

Homburger, H., M. K. Schneider, S. Hilfiker, and A. Lüscher. 2014 Inferring behavioral states of grazing livestock from high-frequency position data alone. PLoS ONE 9:e114522. http://dx.doi. org/10.1371/journal.pone.0114522.

Huzzey, J. M., D. M. Veira, D. M. Weary, and M. A. G. Von Keyserlingk. 2007. Prepartum behavior and dry matter intake identify dairy cows at risk for metritis. J. Dairy Sci. 90:3220-3233. http:// dx.doi.org/10.3168/jds.2006-807.

ION. 1997. ION STD 101: Recommended Test Procedures for GPS Receivers. Institute of Navigation Standards, Alexandria, VA.

Itle, A. J., J. M. Huzzey, D. M. Weary, and M. A. G. von Keyserlingk. 2015. Clinical ketosis and standing behavior in transition cows. J. Dairy Sci. 98:128-134. http://dx.doi.org/10.3168/jds.2014-7932.

Japkowicz, N., and S. Stephen. 2002. The class imbalance problem: A systematic study. Intell. Data Anal. 6:429-449.

John, G. H., and P. Langley. 1995. Estimating Continuous Distributions in Bayesian Classifiers. Pages 338-345 in Eleventh Conf. Uncertainty in Artificial Intelligence, San Mateo. Morgan Kaufmann Publishers, Burlington, MA.

Kilgour, R. J. 2012. In pursuit of "normal": A review of the behavior of cattle at pasture. Appl. Anim. Behav. Sci. 138:1-11. http://dx.doi. org/10.1016/j.applanim.2011.12.002.

Kohavi, R. 1995. A study of cross-validation and bootstrap for accuracy estimation and model selection. Pages 1137-1145 in Proc. Int. Joint Conf. Artificial Intelligence (IJCAI), Montreal, Quebec, Canada, August 20-25. Morgan Kaufmann Publishers, Burlington, MA.

Lewis, J. S., J. L. Rachlow, E. O. Garton, and L. A. Vierling. 2007. Effects of habitat on GPS collar performance: Using data screening to reduce location error. J. Appl. Ecol. 44:663-671. http://dx.doi. org $/ 10.1111 / \mathrm{j} .1365-2664.2007 .01286 . x$

Manske, T., J. Hultgren, and C. Bergsten. 2002. Prevalence and interrelationships of hoof lesions and lameness in Swedish dairy cows. Prev. Vet. Med. 54:247-263. http://dx.doi.org/10.1016/S01675877(02)00018-1.

Munksgaard, L., C. Reenan, and R. Boyce. 2006. Automatic monitoring of lying, standing and walking behavior in dairy cattle. J. Dairy Sci. 89(Suppl. 89):304. (Abstr.)

Nielsen, L. R., A. R. Pedersen, M. S. Herskin, and L. Munksgaard 2010. Quantifying walking and standing behavior of dairy cows using a moving average based on output from an accelerometer. Appl. Anim. Behav. Sci. 127:12-19. http://dx.doi.org/10.1016/j. applanim.2010.08.004.

Nielsen, P. P. 2013. Automatic registration of grazing behavior in dairy cows using 3D activity loggers. Appl. Anim. Behav. 148:179-184. http://dx.doi.org/10.1016/j.applanim.2013.09.001.

Ortiz-Pelaez, Á., and D. U. Pfeiffer. 2008. Use of data mining techniques to investigate disease risk classification as a proxy for compromised biosecurity of cattle herds in Wales. BMC Vet. Res. 4:24. http://dx.doi.org/10.1186/1746-6148-4-24.

Pépin, D., C. Adrados, C. Mann, and G. Janeau. 2004. Assessing real daily distance travelled by ungulates using differential GPS locations. J. Mammal. 85:774-780.

Pluk, A., C. Bahr, A. Poursaberi, W. Maertens, A. Van Nuffel, and D. Berckmans. 2012. Automatic measurement of touch and release angles of the fetlock joint for lameness detection in dairy cattle using vision techniques. J. Dairy Sci. 95:1738-1748. http://dx.doi. org/10.3168/jds.2011-4547.

Quinlan, R. 1993. C4.5: Programs for Machine Learning. Morgan Kaufmann Publishers, San Mateo, CA. 
Reader, J. D., M. J. Green, J. Kaler, S. A. Mason, and L. E. Green. 2011. Effect of mobility score on milk yield and activity in dairy cattle. J. Dairy Sci. 94:5045-5052. http://dx.doi.org/10.3168/ jds.2011-4415.

Robert, B., B. J. Whit, D. G. Renter, and R. L. Larson. 2009. Evaluation of three-dimensional accelerometers to monitor and classify behavior patterns in cattle. Comput. Elect. Agric. 67:80-84. http://dx.doi.org/10.1016/j.compag.2009.03.002.

Roelofs, J. B., F. J. van Eerdenburg, N. M. Soede, and B. Kemp. 2005. Pedometer readings for estrous detection and as predictor for time of ovulation in dairy cattle. Theriogenology 64:1690-1703. http:// dx.doi.org/10.1016/j.theriogenology.2005.04.004.

Sepúlveda-Varas, P., D. M. Weary, and M. A. G. von Keyserlingk. 2014. Lying behavior and postpartum health status in grazing dairy cows. J. Dairy Sci. 97:6334-6343. http://dx.doi.org/10.3168/ jds.2014-8357.

Shahinfar, S., D. Page, J. Guenther, V. Cabrera, P. Fricke, and K. Weigel. 2014. Prediction of insemination outcomes in Holstein dairy cattle using alternative machine learning algorithms. J. Dairy Sci. 97:731-742. http://dx.doi.org/10.3168/jds.2013-6693.

Silper, B. F., I. Robles, A. M. L. Madureira, T. A. Burnett, M. M. Reis, A. M. de Passillé, and R. L. A. Cerri. 2015. Automated and visual measurements of estrous behavior and their sources of variation in Holstein heifers. I: Walking activity and behavior fre- quency. Theriogenology 84:312-320. http://dx.doi.org/10.1016/j. theriogenology.2014.12.029.

Steensels, M., C. Bahr, D. Berckmans, I. Halachmi, A. Antler, and E. Maltz. 2012. Lying patterns of high producing healthy dairy cows after calving in commercial herds as affected by age, environmental conditions and production. Appl. Anim. Behav. Sci. 136:88-95.

Swain, D. L., T. Wark, and G. J. Bishop-Hurley. 2008. Using high fix rate GPS data to determine the relationships between fix rate, prediction errors and patch selection. Ecol. Modell. 212:273-279. http://dx.doi.org/10.1016/j.ecolmodel.2007.10.027.

Van Nuffel, A.. W. Saeys, B. Sonck, J. Vangeyte, K. C. Mertens, B. De Ketelaere, and S. Van Weyenberg. 2015. Variables of gait inconsistency outperform basic gait variables in detecting mildly lame cows. Livest. Sci. 177:125-131. http://dx.doi.org/10.1016/j. livsci.2015.04.008.

Viazzi, S., C. Bahr, T. Van Hertem, A. Schlageter-Tello, C. E. B. Romanini, I. Halachmi, and D. Berckmans. 2014. Comparison of a three-dimensional and two-dimensional camera system for automated measurement of back posture in dairy cows. Comput. Elect. Agric. 100:139-147. http://dx.doi.org/10.3168/jds.2012-5806.

Whay, H. R., D. C. J. Main, L. E. Green, and A. J. F. Webster. 2003. Assessment of the welfare of dairy cattle using animal-based measurements: Direct observations and investigation of farm records. Vet. Rec. 153:197-202. http://dx.doi.org/10.1136/vr.153.7.197. 